For oral presentation in Session "MesoFracture Mechanics" organized by GC Sih

\title{
Meso-fracture of Metal Matrix Composites Reinforced by Particles of Large Volume Fraction
}

\author{
Wei YANG, Chouwei ZHOU and Daining FANG \\ Department of Engineering Mechanics, Tsinghua University
}

Beijing, 100084, China

\begin{abstract}
Meso-fracture process for metal matrix composites (MMC) reinforced by particles of large volume fraction is pursued in this paper. Particle reinforced $\mathrm{Al}_{2} \mathrm{O}_{3} / \mathrm{Al} \mathrm{MMC}$ is made under a pressure-less filtration technique. Samples are tested in uniaxial tension, compression and three-point bending, and their fracture patterns and fractography are recorded. The micromechanics model of the composite is composed of identical elastic spheres linked by columns of elastic perfectly plastic matrix. The metallic matrix is treated in two categories: the elastic perfectly plastic von Mises columns linking various particles are actively engaged in forming the skeleton of the MMC; while the rest of the matrix is inactive and only serves to constrain the radial deformation of linking columns. The failure of the interface is governed by the interfacial energy release rate, the matrix damage by the Rice-Tracey void evolution law, and the particle breaking by the largest principal stress. The macroscopic stress-strain relation is derived from the normal and tangential moduli of the two-particle linkages by averaging over a statistical assembly of three packing patterns. Two random variables are adopted for the fluctuations of local stresses in particles and along particle/matrix interfaces. The simulated overall constitutive responses agree with the experiments for the uniaxial tensile, uniaxial compression and three-point bending specimens. The simulated meso-fracture patterns under three testing configurations reproduce the experimental results. Simulated proportions of failure by interface debonding, matrix voiding and particle breaking also agree with the experiments.
\end{abstract}

KEY WORDS: meso-fracture, particle reinforcement, metal matrix composite, FEM simulation. 